\title{
Visualização temporal no ensino de história: uma análise gráfica de ferramentas visuais didáticas de representação do tempo
}

\author{
Temporal visualization in teaching history: a graphic analysis of visual \\ didactic tools for representing time
}

Juliana Ferreira de Oliveira, Juliana Bueno

visualização de dados, análise gráfica, história

\begin{abstract}
As demandas informacionais no contexto educacional contemporâneo apresentam novos desafios de articular diferentes linguagens no ensino e aprendizagem. Para a disciplina de História, ferramentas de visualização têm sido importantes meios de diversificar abordagens sobre a informação, algo fomentado pelo currículo brasileiro para o Ensino Médio. Motivado por isso, e pelos diálogos possíveis entre Design da Informação e Educação, esse trabalho busca averiguar as dimensões comuns utilizadas na representação de dados históricos, principalmente temporais, trazidas por artefatos de visualização didática da história. Para tal, foi conduzida uma análise gráfica de artefatos, por meio do modelo de variáveis gráficas de Bertin (1967). No total foram elencadas oito ferramentas impressas e digitais, analisadas a partir de elementos gráficos básicos e variáveis gráficas. Por fim, discute-se as principais tendências trazidas pelos artefatos e como elas se relacionam aos objetivos da visualização da informação, o que fornece embasamento para desenvolvimentos futuros de artefatos semelhantes.
\end{abstract}

data visualization, graphic analysis, history

Informational demands in the contemporary educational context present new challenges in articulating different languages in teaching and learning. For the subject of History, visualization tools have been important means of diversifying approaches to information, something promoted by the Brazilian high school syllabus. Motivated by this, and by the possible dialogues between Information Design and Education, this research aims to investigate the common dimensions used in the representation of historical data, brought by artifacts with didactic visualization of history. To this end, a graphic analysis of artifacts was developed, using Bertin's (1967) model of graphic variables. In total, eight printed and digital tools were listed, analyzed from basic graphic elements and graphic variables. Finally, there is a discussion about the main trends brought by the artifacts, and how they relate to the objectives of data visualization, which provides a basis for future developments of similar artifacts.

\section{Introdução}

O currículo para o Ensino Médio brasileiro busca promover abordagens sistêmicas sobre os conteúdos. Para tal, ressalta a importância de trazer novos recursos e linguagens à sala de aula, a fim de preparar estudantes para a complexidade informacional da contemporaneidade (Brasil, 2018).

Anais do $10^{\circ} \mathrm{CIDI}$ e $10^{\circ} \mathrm{CONGIC}$

Kelli C.A.S. Smythe, Rafael de Castro Andrade (orgs.)

Sociedade Brasileira de Design da Informação - SBDI

Curitiba | Brasil | 2021
Proceedings of the $10^{\text {th }} \mathrm{CIDI}$ and $10^{\text {th }}$ CONGIC

Kelli C.A.S. Smythe, Rafael de Castro Andrade (orgs.)

Sociedade Brasileira de Design da Informação - SBDI Curitiba | Brazil | 2021 
A prática de diversificação de linguagens é familiar à disciplina de História em diferentes níveis do ensino, especialmente no que se refere a determinadas vertentes historiográficas, como a história global. Nessa, a articulação sincrônica da informação gráfica tem ajudado a contornar obstáculos metodológicos do conhecimento histórico (Jordheim, 2017).

Tabelas sincrônicas e linhas do tempo adentram o presente remodelando a representação visual do tempo mecânico e social. A abstração da temporalidade requer a articulação de sistemas de informação em diferentes graus de complexidade, um desafio que tem ajudado a dar forma a novas ferramentas (Pschetz \& Bastian, 2018).

A partir disso, questiona-se quais os parâmetros de design da informação que permitem que a representação visual do tempo se alinhe à prática didática. Partindo dessa problemática, este artigo tem como objetivo investigar as principais dimensões da representação do tempo histórico em artefatos didáticos de visualização. Essa mediação entre sistemas de informação e sala de aula busca expandir a comunicação multidisciplinar do design da informação.

Os resultados parciais da pesquisa integram Trabalho de Conclusão de Curso teórico, na graduação em Design Gráfico da Universidade Federal do Paraná, buscando uma aproximação com História no Ensino Médio, a fim de construir visualizações de dados acessiveis ao contexto escolar.

Para investigar as principais dimensões de representação gráfica da informação, foram elencados artefatos didáticos, os quais foram submetidos à análise gráfica a partir das variáveis gráficas de Bertin (1967). Em seguida, são discutidos os resultados obtidos.

\section{Referencial teórico}

Para o conhecimento histórico, que no Ensino Médio integra o itinerário formativo das Ciências Humanas e Sociais Aplicadas, a aprendizagem está sujeita ao desenvolvimento de competências de análise, comparação, interpretação etc. Para a Base Nacional Comum Curricular, as linguagens (e.g. gráfica, cartográfica) utilizadas para desenvolvê-las têm importância equivalente (Brasil, 2018).

O avanço tecnológico contemporâneo tem proporcionado múltiplas formas de buscar em tais linguagens novas formas de apresentação do tempo. Isso desperta transformações significativas em práticas de visualização, como da história global.

A história global busca a sincronização de cronologias globais como artifício de comparação analítica (Jordheim, 2010). Desde a Idade Moderna, esse caráter comparativo encontra suporte ferramental em modelos de visualização da informação, como tabelas sincrônicas e linhas do tempo. Entretanto, o mundo globalizado desperta na história global uma adequação social (Adelman, 2017), enquanto demanda novos formatos de apresentação, mais tecnológicos e expansivos.

Os tradicionais modelos sincrônicos de Gatterer (de 1766) e de Strass (de 1804) são exemplos emblemáticos da historiografia visual de seus contextos, que se modificaram profundamente nos séculos seguintes. Nesses, civilizações ocupam o papel ao longo de linhas 
do tempo verticais, assumindo formas retangulares ou orgânicas que se interferem simbolicamente, acompanhados de cores codificadas que representam identidade cultural e relevância geopolítica (Araújo, 2015; Jordheim, 2017).

Mesmo distanciados por quase dois séculos desses autores, as publicações impressas The Timetables of History (1946) e Timetables of History (1999) apostam em modelos tradicionais em matriz. Neles, a narrativa verbal assume importância primária.

Já os artefatos digitais - Geacron (2011), Chronozoom (2012), World Population History (2015) e Ostellus (2020) - ilustram as ambições da sincronização temporal do século XXI. A interface, por sua vez, proporciona recursos de interatividade que mudam a forma como a informação é disponibilizada.

As inovações individuais trazidas por cada uma dessas ferramentas contemplam também novas dimensões de representação - dimensões essas que permeiam os objetivos educativos e informacionais de design. Assim, mapear os elementos e suas relações semânticas oferece uma base comparativa para compreender como tais formas de representação temporal vêm sendo moldadas.

O modelo de variáveis gráficas de Bertin (1967), original da cartografia e revisado seguidamente por diversos autores (Engelhardt, 2002; Mijksenaar, 1997; Horn, 1998), fornece uma base sólida para esse mapeio, a partir das complementações de Meirelles (2013) - cuja organização bibliográfica e metodologia direcionaram o desenvolvimento da pesquisa na qual este trabalho se insere. $\mathrm{O}$ autor propõe uma estruturação da linguagem gráfica a partir de três elementos gráficos (Meirelles, 2013):

- Ponto: adimensional, demarca localização;

- Linha: unidimensional, ilustra direção e distância;

- Plano: bidimensional, delimita espaço e demonstra escala.

Dentro da representação, esses elementos se diversificam em formas de expressão particulares à cognição, denominadas variáveis gráficas:

- Posição;

- Tamanho;

- Valor;

- Textura;

- Cor;

- Orientação;

- Forma.

Depois, cada variável é associada a propriedades significadoras, que representam a semântica dentro do todo:

- Quantitativa, quando permitem estimar diferenças quantitativas;

- Ordenada, quando expressam gradação visual e ordem; 
- Seletiva, quando permitem selecionar elementos frente a um todo;

- Associativa, quando propiciam agrupamento;

- Dissociativa, quando alteram a visibilidade;

\section{Metodologia}

Figura 1: Etapas da pesquisa. Fonte: as autoras.

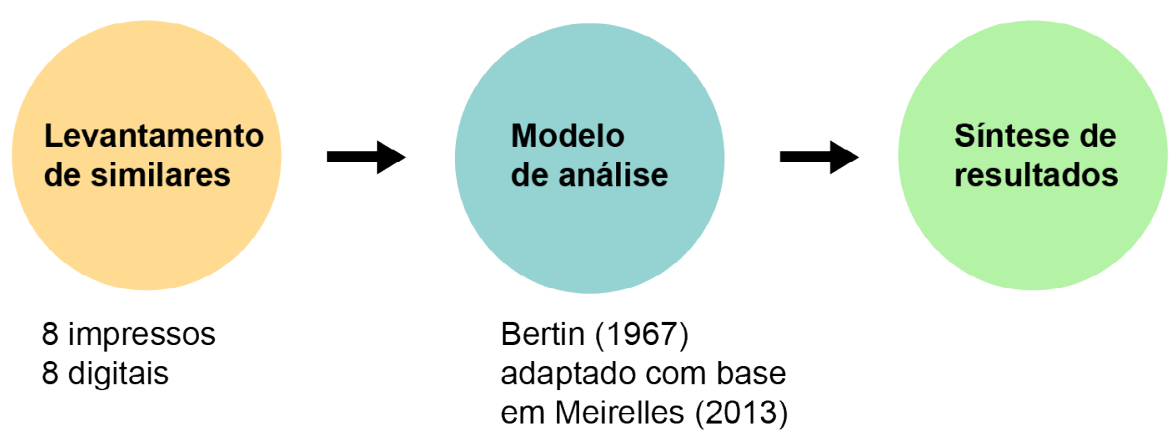

Para elencar as principais tendências nas dimensões da representação histórica, primeiramente foram selecionados artefatos didáticos a partir de revisão bibliográfica sobre história e visualização do tempo. As amostras coletadas foram selecionadas dentre ferramentas que trouxeram contribuições à prática de sincronização de eventos no tempo durante diferentes contextos históricos (conforme trazido por Jordheim, 2014; Araújo, 2015; Grafton \& Rosenberg, 2010; Psyshkin \& Bogdanov, 2014). Além dos direcionamentos da bibliografia, optou-se por amostras de diferentes contextos históricos, a fim de investigar mudanças de representação ocasionadas por novas demandas de informação e tecnologias. Para contexto, foram empregadas as seguintes estratégias:

- Séculos XVIII e XIX: exemplares (1 e 2) que romperam com modelos verbais, recorrendo a artifícios esquemáticos e pictóricos;

- Século XX: exemplares ( 3 e 4 ) que revitalizaram a prática de tabelas sincrônicas, em contraste aos do século XVIII;

- Século XXI: exemplares (5, 6, 7 e 8) com diferentes abordagens sobre representação do tempo e espaço, que retomam tanto práticas visuais do século XVIII e XX.

Ao todo, foram coletadas oito ferramentas, que têm como objetivo principal a visualização temporal (podendo abranger ou não a dimensão espacial). Quatro delas eram impressas e quatro eram digitais, desses quais apenas três apresentavam visualização espacial cartográfica (Figura 2). 
Figura 2: Artefatos selecionados. Fonte: as autoras.

\section{Impressos}

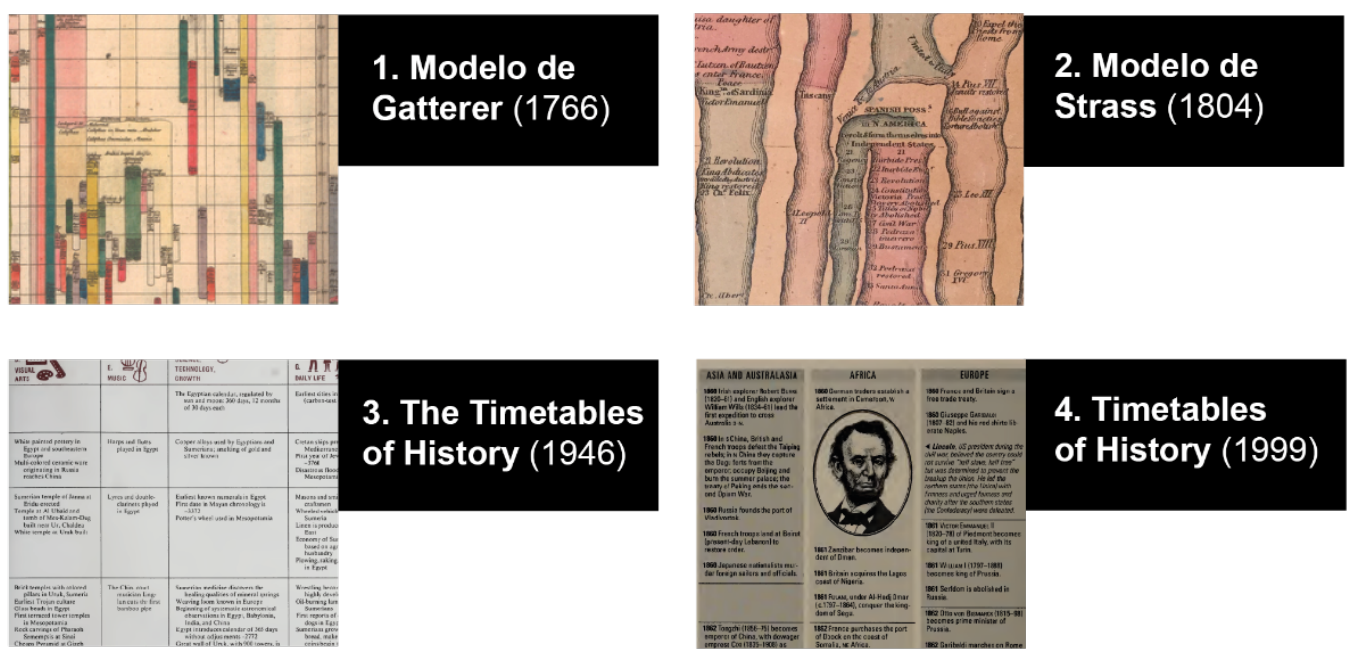

Digitais
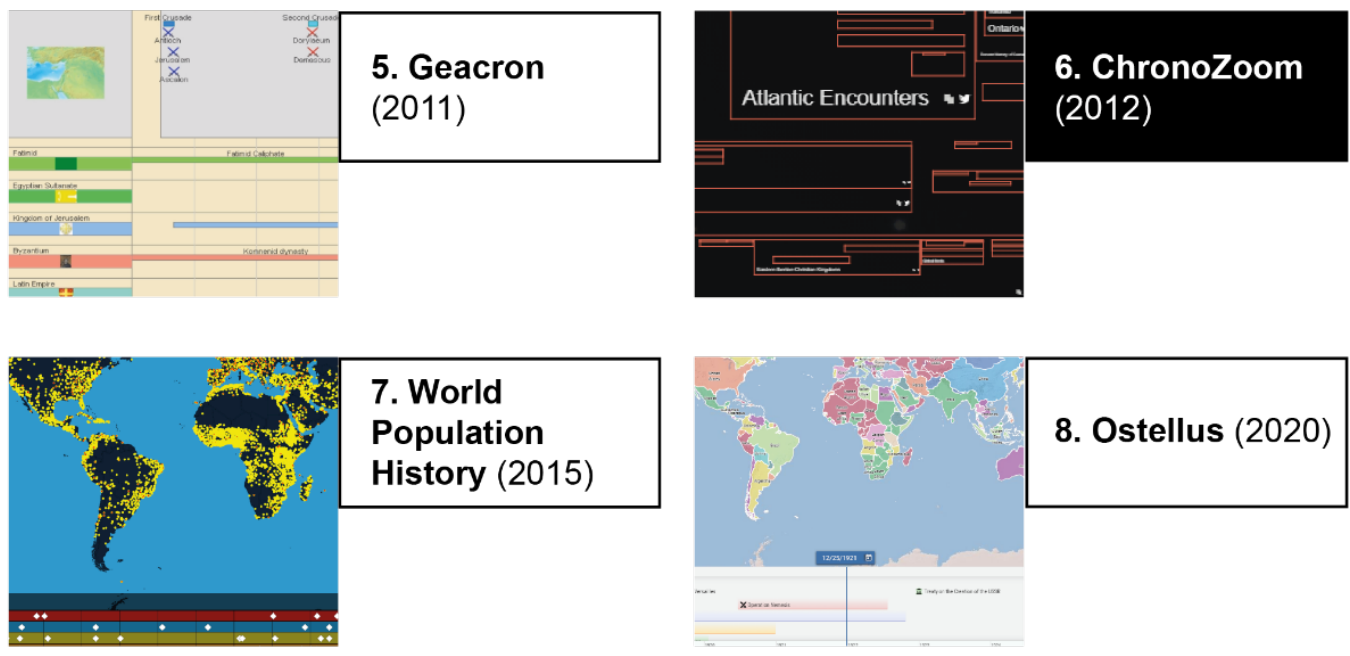

\section{World Population History (2015)}

\section{Legenda}

Linha do tempo

Linha do tempo e mapa

Em seguida, o modelo de variáveis gráficas de Bertin foi complementado a partir de contribuições de autores diversos (Meirelles, 2013). Além das variáveis propostas originalmente, foram adicionadas saturação, matiz, transparência e disposição. A figura 3 traz as variáveis presentes no modelo e a eficiência com a qual podem despertar determinadas propriedades significadoras, conforme a bibliografia (Meirelles, 2013). 
Figura 3: Relação de variáveis e suas propriedades significadoras. Variáveis adicionais se encontram na coluna à direita. Fonte: as autoras, baseado em Meirelles (2013).

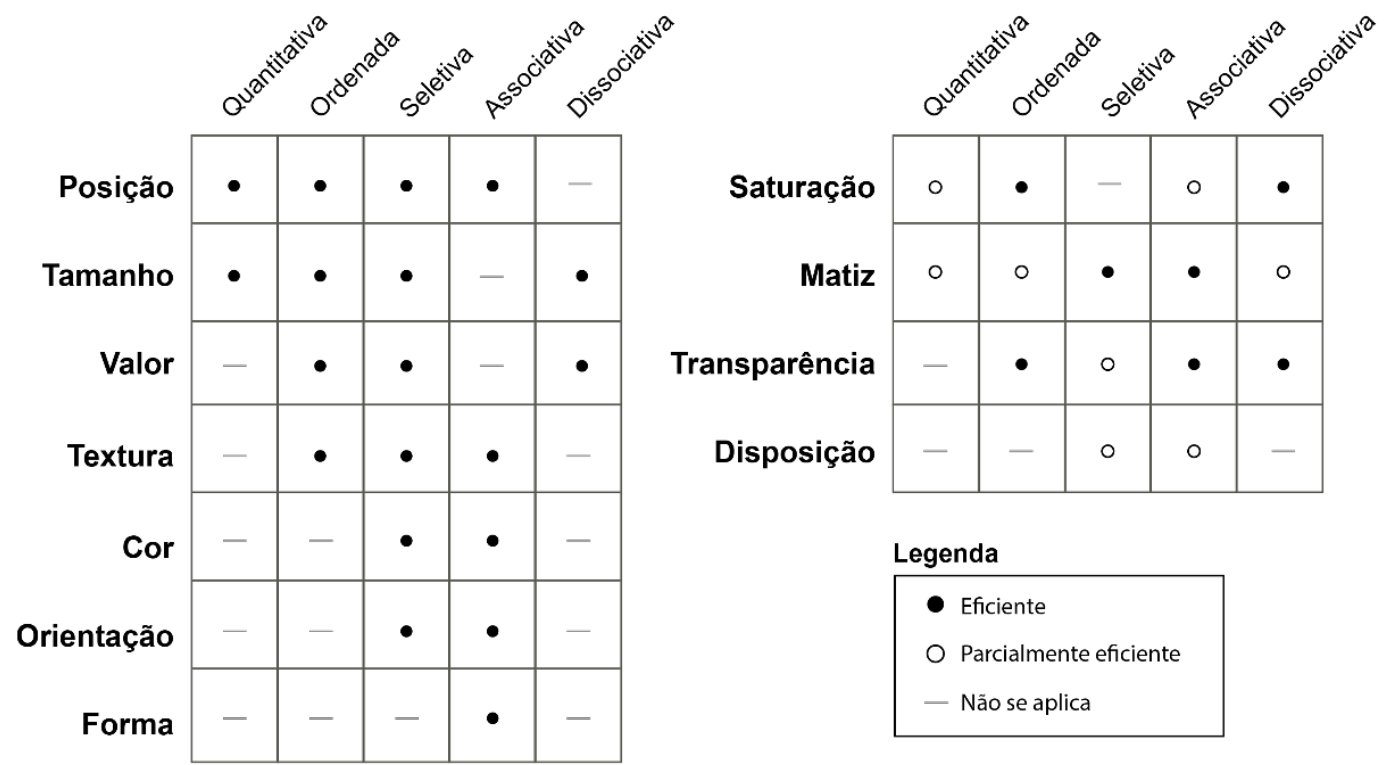

Cada similar foi avaliado individualmente a partir das variáveis gráficas no modelo, momento esse em que se buscou apontar a presença ou não delas a partir da variação visual encontrada nos elementos ponto, linha e área. Entretanto, considerando que três artefatos continham simultaneamente visualização temporal e espacial (mapa), com linguagens distintas entre si, optou-se por analisar as partes individualmente. Assim, foram geradas duas tabelas de síntese principais.

Ao fim, agruparam-se tabelas no intuito de compilar a quantidade de ocorrências de elementos atrelados a cada variável, elencando assim as principais tendências presentes nos artefatos. Paralelamente, apenas no recorte da análise temporal, os resultados foram agrupados em outras duas tabelas, sendo uma para impressos e outra para digitais, para comparação entre tendências de cada formato.

\section{Resultados e discussão}

A síntese dos resultados obtidos mostra um leve desvio de tendências entre representações temporais e espaciais no que se refere ao uso de elementos gráficos predominantes. Enquanto linhas do tempo apresentam mais uso de linha e área, mapas trazem ponto e área.

É possível notar que ambos os formatos de visualização da informação apresentam as mesmas variáveis gráficas predominantes: posição, tamanho, cor, matiz e disposição. Também é possível observar um equilíbrio no uso de propriedades significadoras (Figura 4), e todas as variáveis apresentam eficiente ou parcialmente propriedades seletivas. 
Figura 4: Relação de variáveis mais presentes e suas propriedades significadoras. Fonte: as autoras.

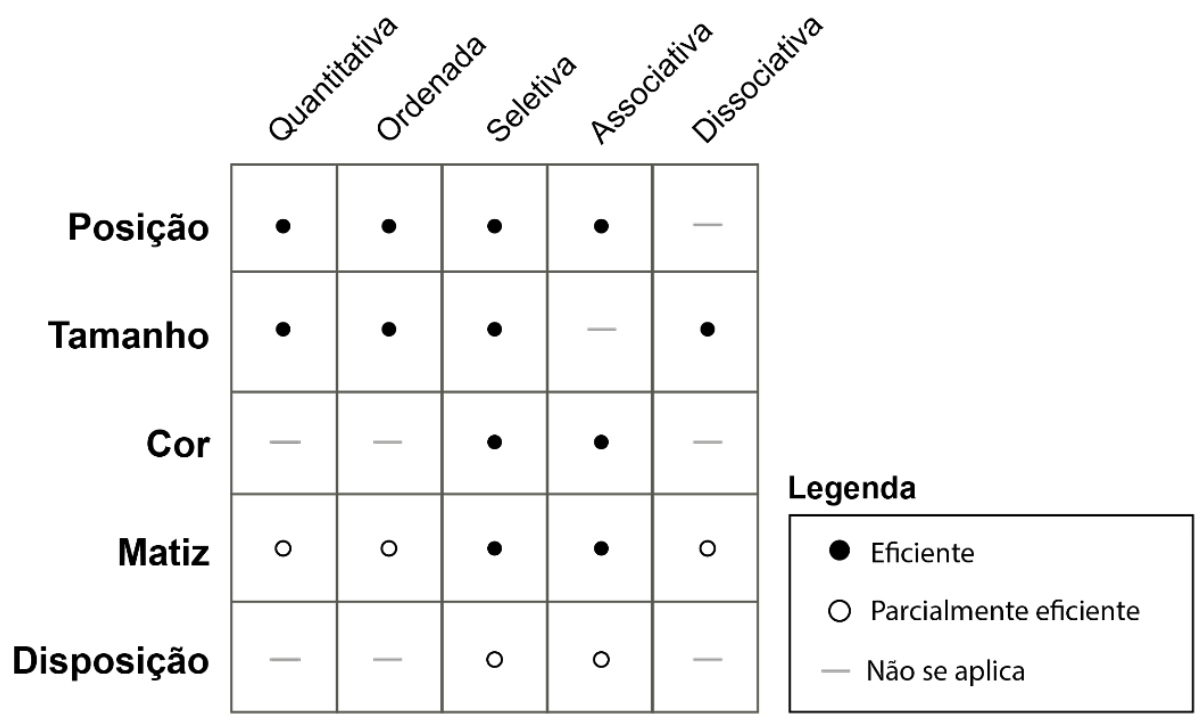

Ao comparar os resultados obtidos com linhas do tempo em impressos (todos lançados anteriormente ao século XXI) com os digitais (lançados depois de 2010), é possível notar uma tendência na expansão da complexidade de representação. Artefatos digitais tendem a apresentar maior variedade de uso de elementos, principalmente pontos, e de variáveis gráficas em geral (as mesmas expressas na Figura 4, com adição de "valor").

Para artefatos impressos, apesar da quantidade menor de variáveis, nota-se uma preocupação em trazer aquelas com potencial para assumir mais propriedades significadoras. Ambas as variáveis mais presentes - posição e tamanho - contemplam quatro propriedades, de forma complementar.

A maior diversidade no uso de elementos e variáveis nos recursos digitais demonstra as possibilidades na expansão da visualização que surge ao aliar a informação a recursos tecnológicos. Essa diversidade possibilita a incorporação de novos códigos e linguagens à apresentação do conhecimento histórico, e caminhos possíveis para que o ensino alcance diferentes públicos e abordagens.

\section{Considerações finais}

Operar com sistemas de informação contemporâneos traz novos desafios para a educação hoje. A complexidade do tempo histórico e de suas implicações sociais torna propício buscar artifícios experimentais de visualização, para torná-la mais intuitiva.

Diferentes elementos, variáveis e propriedades emergem das relações entre informação e temporalidade. Para o presente, diversificar as linguagens implica em explorar novas dimensões de representação, cabíveis às tecnologias disponíveis.

Por fim, os resultados levantados são fundamentais para pautar as etapas seguintes da pesquisa teórica, oferecendo diretrizes para a delimitação metodológica de novos recursos 
didáticos de visualização, compatíveis com as demandas do presente. Ressalta-se, ainda, a possibilidade de expandir os estudos para recursos contemporâneos analógicos e colaborativos.

\section{Referências}

Araújo, A. M. (2015). Imagens da simultaneidade e os impasses da narrativa - O caso da Synopsis historiae universalis (1766) de Johann Christoph Gatterer. Tempo, 21 (38), 192215. https://doi.org/10.1590/tem-1980-542x2015v213801

Adelman, J. (2017). What is global history now? Aeon. Disponível em https://aeon.co/essays/isglobal-history-still-possible-or-has-it-had-its-moment

Bertin, J. (1967) Semiology of Graphics: Diagrams, Networks, Maps. Redlands: ESRI Press.

Brasil. (2018). Base Nacional Comum Curricular. Ministério da Educação. Disponível em http://basenacionalcomum.mec.gov.br/abase/

Engelhardt, Y. (2002). The Language of Graphics: A Framework for the Analysis of Syntax and Meaning in Maps, Charts and Diagrams. ILLC dissertation series.

Grafton, A., \& Rosenberg, D. (2010). Cartographies of Time ( $1^{\mathrm{a}}$ ed.). Princeton Architectural Press.

Horn, R. E. (1998). Visual Language: Global Communication for the 21st Century (1st ed.). MacroVU Press.

Jordheim, H. (2017). Synchronizing the World: synchronism as historiographical practice, then and now. History Of The Present, 7 (1), 59-95.

https://doi.org/10.5406/historypresent.7.1.0059

Jordheim, H. (2014). Introduction: Multiple Times and the Work of Synchronization. History and Theory, 53 (4), 498-518. https://doi.org/10.1111/hith.10728

Meirelles, I. (2013) Design for Information: an introduction to the histories, theories, and the best practices behind effective information visualizations. Massachusetts: Rockport.

Mijksenaar, P. (1997). Visual function: an introduction to information design. Rotterdam: 010 Publishers.

Pschetz, L., \& Bastian, M. (2018). Temporal Design: rethinking time in design. Design Studies, 56, 169-184. https://doi.org/10.1016/j.destud.2017.10.007.

Pyshkin, E., \& Bogdanov, N. (2014). Learning History with Timelines: Use Cases, Requirements and Design. IEEE. https://doi.org/10.15439/2014f106

\section{Sobre as autoras}

Juliana Ferreira de Oliveira, graduanda, UFPR, Brasil <jubrotx@gmail.com> Juliana Bueno, Dra. e professora, UFPR, Brasil, <julianabueno.ufpr@gmail.com> 